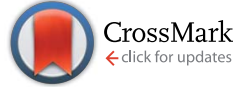

Cite this: RSC Adv., 2017, 7, 13191

Received 28th November 2016 Accepted 13th February 2017

DOI: $10.1039 / c 6 r a 27449 h$

rsc.li/rsc-advances

\title{
Understanding the emergence of collective motion of microtubules driven by kinesins: role of concentration of microtubules and depletion force $\dagger$
}

\author{
Ai Saito, $\neq^{\mathrm{a}}$ Tamanna Ishrat Farhana, $\$^{\mathrm{a}}$ Arif Md. Rashedul Kabir, ${ }^{\mathrm{b}}$ Daisuke Inoue, ${ }^{\mathrm{b}}$ \\ Akihiko Konagaya, ${ }^{c}$ Kazuki Sada ${ }^{a b}$ and Akira Kakugo*ab
}

\begin{abstract}
We investigated the role of the concentration of microtubules and depletion force in the emergence of collective motion of microtubules driven by kinesins. Critical concentrations were found for both microtubules and the depletant to demonstrate the collective motion of microtubules. More importantly, the kinetics of the collective motion were found to be significantly dependent on the concentration of microtubules and the depletant. Substantial variations in the time course of emergence of the collective motion of microtubules were observed in response to the changes in concentration of the microtubules and the depletant. This study provides new insights into the kinetics of the collective motion of microtubules driven by kinesins, which might also be useful in understanding the coordinated behavior of other self-propelled systems.
\end{abstract}

\section{Introduction}

Collective motion is a fascinating display of coordinated behavior of moving or self-propelled objects. ${ }^{1-3}$ A wide range of self-propelled objects, such as animals, birds, cells, and bacteria, exhibit collective motion in nature, which is most often associated with the formation of fascinating large scale patterns. $^{4-10}$ In silico studies significantly contributed to much of our understanding of the coordinated behavior and pattern formation of the self-propelled objects. ${ }^{11-18}$ A suitable experimental system has long been awaited for demonstrating the collective motion in vitro in order to verify the mechanisms of collective motion and pattern formation suggested by the in silico studies. In recent years, the biomolecular motor systems F-actin/myosin and microtubule/dynein have emerged as ideal candidates for experimentally demonstrating the collective motion through an in vitro gliding assay. ${ }^{\mathbf{1 9 , 2 0}}$ At the same time

${ }^{a}$ Graduate School of Chemical Sciences and Engineering, Hokkaido University, Sapporo 060-0810, Hokkaido, Japan. E-mail: kakugo@sci.hokudai.ac.jp; Fax: +8111-706-3474; Tel: +81-11-706-3474

${ }^{b}$ Faculty of Science, Hokkaido University, Sapporo 060-0810, Hokkaido, Japan ${ }^{c}$ School of Computing, Tokyo Institute of Technology, Yokohama, 226-8501, Japan

$\dagger$ Electronic supplementary information (ESI) available: Supporting Fig. (1-5) showing the orientation of microtubules in the absence of the depletant, effect of methylcellulose concentration on depletion force, snuggling probability and velocity of gliding microtubules, and buffer viscosity. Supporting movie 1 shows the effect of microtubules and depletant (methylcellulose) concentration on the emergence of collective motion (the movie time is accelerated 100-fold i.e., $1 \mathrm{~s}$ of movie time equals $100 \mathrm{~s}$ of experimental time). See DOI: 10.1039/c6ra27449h \$ These authors contributed equally to this work. the failure in demonstrating the collective motion of microtubules driven by kinesins, the most widely used biomolecular motor in vitro, raised a question about the universality of biomolecular motor systems to demonstrate collective motion. Although formation of an active gel of microtubules crosslinked via kinesin clusters has been reported, ${ }^{21}$ it was not sufficient to understand the coordinated behavior of kinesin driven microtubules using the in vitro gliding assay as demonstrated for other biomolecular motor systems. Recently, we succeeded in demonstrating the collective motion of the microtubules driven by kinesins, which was associated with a transition in the orientation of the microtubules from an isotropic to a nematic phase, leading to the formation of fascinating stream patterns. ${ }^{22}$ We showed that mutual interaction among the microtubules, which could be regulated by employing a depletion force among the microtubules, is an important criterion for demonstrating the collective motion of the microtubules on kinesins. ${ }^{22}$ Nonetheless, how different parameters influence the emergence of collective motion of the kinesin driven microtubules has not been well understood since the role of different parameters in the collective motion of microtubules has not been investigated. In the present study, by examining the effects of the concentration of microtubules and a depletant (methylcellulose), we explored in detail the time course of the emergence of collective motion (i.e., kinetics of transition of microtubule's orientation from an isotropic to a nematic phase). We found that the concentration of the microtubules and methylcellulose had a profound influence on the kinetics of emergence of collective motion of the kinesin 
driven microtubules. High concentrations of microtubules or methylcellulose can substantially accelerate the phase transition of microtubules (i.e. emergence of the collective motion of microtubules). Moreover, a minimal concentration of both of the microtubules and methylcellulose was required to demonstrate the collective motion. The minimal concentration of microtubules was altered when the concentration of methylcellulose employed in the gliding assay was varied and vice versa. This study provided a comprehensive scenario of the roles of microtubule concentration and depletion force on the kinetics of the collective motion of microtubules driven by kinesins, which consequently would help obtain a better understanding of the coordinated behavior of other selfpropelled objects. ${ }^{23-25}$

\section{Experimental}

\section{Preparation and labelling of proteins}

Tubulin was purified from porcine brain using a high concentration PIPES buffer (1 M PIPES, $20 \mathrm{mM}$ EGTA, and $10 \mathrm{mM}$ $\mathrm{MgCl}_{2} ; \mathrm{pH}$ was adjusted to 6.8 using $\left.\mathrm{KOH}\right){ }^{26}$ The high concentration PIPES buffer and BRB80 buffer were prepared using PIPES from Sigma, and the $\mathrm{pH}$ was adjusted using $\mathrm{KOH}$. Kinesin- 1 consisting of the first 575 amino acid residues of human kinesin-1 was prepared by partially modifying the expression and purification methods described in the literature. ${ }^{27}$ Rhodamine-labelled microtubules were obtained by polymerizing a mixture of rhodamine-labelled tubulin (RT) and non-labelled tubulin (WT) (RT:WT $=4: 1$; final tubulin concentration, $55.6 \mu \mathrm{M}$ ) at $37{ }^{\circ} \mathrm{C}$ in the presence of $1 \mathrm{mM}$ GTP according to the standard technique. The solution containing the microtubules was then diluted with motility buffer $(80 \mathrm{mM}$ PIPES, $1 \mathrm{mM}$ EGTA, $2 \mathrm{mM} \mathrm{MgCl}_{2}, 0.5 \mathrm{mg} \mathrm{mL}{ }^{-1}$ casein, $1 \mathrm{mM}$ DTT, $10 \mu \mathrm{M}$ paclitaxel and $1 \%$ DMSO; pH 6.8).

\section{In vitro gliding assay of microtubules}

A flow cell with dimensions of $5.0 \times 5.0 \times 0.15 \mathrm{~mm}^{3}(w \times l \times h)$ was assembled from two cover glasses of sizes $(5 \times 7) \mathrm{mm}^{2}$ and $(40 \times 50) \mathrm{mm}^{2}$ (MATSUNAMI), and a double-sided tape was used as the spacer. First, the surface of the flow cell was coated with casein buffer ( $80 \mathrm{mM}$ PIPES, $1 \mathrm{mM}$ EGTA, $1 \mathrm{mM} \mathrm{MgCl}$ and $\sim 0.5 \mathrm{mg} \mathrm{mL}^{-1}$ casein; $\mathrm{pH} 6.8$ ) and incubated for $3 \mathrm{~min}$. Then, 3 $\mu \mathrm{L}$ of kinesin-1 solution ( $\sim 80 \mathrm{mM}$ PIPES, $1 \mathrm{mM}$ EGTA, $1 \mathrm{mM}$ $\mathrm{MgCl}_{2}, 0.5 \mathrm{mg} \mathrm{mL}{ }^{-1}$ casein, $1 \mathrm{mM}$ DTT, $10 \mu \mathrm{M}$ paclitaxel/DMSO, $\sim 1 \%$ DMSO; $\mathrm{pH}$ 6.8) was applied to the flow cell and incubated for $1 \mathrm{~min}$ to bind the kinesin to the casein coated surface of the flow cell through non-specific interaction. The flow cell was washed with $5 \mu \mathrm{L}$ of motility buffer ( $80 \mathrm{mM}$ PIPES, $1 \mathrm{mM}$ EGTA, $1 \mathrm{mM} \mathrm{MgCl}_{2}, 0.5 \mathrm{mg} \mathrm{mL}^{-1}$ casein, $1 \mathrm{mM}$ DTT, $10 \mathrm{mM}$ paclitaxel and $\sim 1 \%$ DMSO; pH 6.8). Next, $3 \mu \mathrm{L}$ of microtubule solution of a prescribed concentration was applied to the flow cell and incubated for $2 \mathrm{~min}$, followed by washing with $5.0 \mu \mathrm{L}$ of motility buffer. Then, $10 \mu \mathrm{L}$ of $10 \mathrm{mM}$ adenosine triphosphate (ATP) buffer containing methylcellulose of a prescribed concentration ( 80 mM PIPES, $1 \mathrm{mM}$ EGTA, $1 \mathrm{mM} \mathrm{MgCl}_{2}, 0.5 \mathrm{mg} \mathrm{mL}^{-1}$ casein, $1 \mathrm{mM}$ DTT, $10 \mu \mathrm{M}$ paclitaxel/DMSO, 0-0.3 wt\% methylcellulose, $\sim 1 \%$ DMSO; pH 6.8) was added to the flow cell. The flow cell was placed inside the inert chamber after the addition of ATP, and humid nitrogen gas was passed through the chamber to remove oxygen from the chamber. ${ }^{28,29}$ The time of ATP addition was set at $0 \mathrm{~min}$, and microtubules were monitored with fluorescence microscopy. All these experiments were performed at room temperature.

\section{Quantitative analysis of collective motion of microtubules}

The orientation of microtubules was calculated in terms of the nematic order parameter $(S)$, which was calculated according to the following equation ${ }^{30,31}$ using ImageJ plugin, orientation $\mathrm{J}$ (http://bigwww.epfl.ch/demo/orientation/):

$$
S=\frac{1}{N} \sqrt{\left(\sum_{i=0}^{180} R_{i} \cos 2 \Theta_{i}\right)^{2}+\left(\sum_{i=0}^{180} R_{i} \sin 2 \Theta_{i}\right)^{2}}
$$

where $N$ refers to total number of microtubules in the frame and $R_{i}$ is frequency of the angle of individual microtubules $\Theta_{i}(i=$ 0 to 180). The range of $\Theta_{i}$ was fixed from $0^{\circ}$ to $180^{\circ}$ based on the symmetry of horizontally or vertically aligned microtubules with respect to the $X$-axis. Since the microtubules in the nematic phase were oriented along the $X$-axis, we fixed the director for both the isotropic and nematic phase along the $X$-axis and measured the nematic order parameter of microtubules in the $X Y$ plane.

\section{Microscopic image capture}

The samples were illuminated with a $100 \mathrm{~W}$ mercury lamp and visualised with an epi-fluorescence microscope (Eclipse Ti; Nikon) using an oil-coupled Plan Apo $60 \times 1.40$ objective (Nikon). Filter blocks with UV-cut-off specifications (TRITC: EX540/25, DM565, BA606/55; GFP-HQ: EX455-485, DM495, BA500-545; Nikon) were used in the optical path of the microscope to allow the visualisation of samples while eliminating the UV portion of the radiation and minimising the harmful effects of UV radiation on the samples. Images and movies were captured using a cooled CMOS camera (Neo sCMOS; Andor) connected to a PC. To capture a field of view for more than several minutes, ND filters (ND4, 25\% transmittance) were inserted into the illuminating light path of the fluorescence microscope to avoid photobleaching.

\section{Results and discussion}

To explore the kinetics of the collective motion of microtubules driven by kinesins, we investigated in detail the effect of some relevant parameters, such as the concentration of microtubules and a depletant (methylcellulose), on the collective motion. We performed an in vitro gliding assay of microtubules on kinesins by varying the concentration of the microtubules and the methylcellulose in the assay system (Fig. 1A). Microtubules were prepared through polymerization of fluorescence dye labeled tubulins (molecular weight $\sim 55 \mathrm{kDa}$ ) and stabilized with Taxol, as mentioned in the experimental section. Due to the stochastic assembly process, the length of the microtubules varied over 

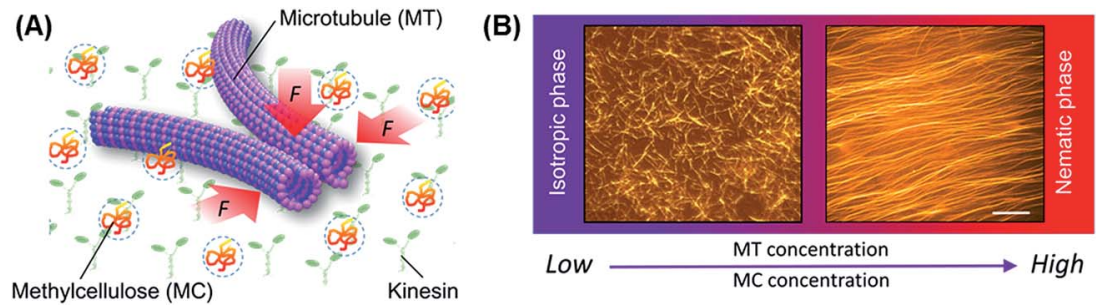

Fig. 1 In vitro gliding assay of microtubules on a kinesin coated surface in the presence of methylcellulose. (A) Schematic diagram of in vitro gliding assay. (B) Effect of concentration of microtubules and depletant (methylcellulose) on the organization of microtubules, where left and right side images show the orientation of $1.0 \mu \mathrm{M}$ microtubules in the absence of methylcellulose and $10 \mu \mathrm{M}$ microtubules in the presence of 0.3 wt\% methylcellulose, respectively. Scale bar: $20 \mu \mathrm{m}$.

a wide range. We calculated the number average length $\left(\left\langle L_{n}\right\rangle\right)$ and weight-average length $\left(\left\langle L_{\mathrm{w}}\right\rangle\right)$ of microtubules as $15.23 \mu \mathrm{m}$ and $16.69 \mu \mathrm{m}$, respectively (number of microtubules considered: 100), from which the polydispersity index $\left(L_{\mathrm{w}} / L_{\mathrm{n}}\right)$ was found to be $\sim 1.1 .{ }^{32}$

As the first step, we performed the gliding assay of microtubules on the kinesin coated substrate in the absence of the depletant varying the concentration of the microtubules over a wide range (1-15 $\mu \mathrm{M})$. The gliding assay of microtubules was performed on a flow cell made from cover glass (see experimental section). In brief, recombinant kinesins consisting of 575 amino acid residues of human kinesin-1 were adsorbed to the casein coated surface of the flow cell through a non-specific interaction. Next, the fluorescence dye-labeled microtubules with length of $15.78 \pm 2.29 \mu \mathrm{m}$ (average \pm standard deviation) were deposited to the flow cell through interactions with the kinesins. By adding adenosine triphosphate (ATP), motility of the microtubules was initiated on the kinesin carpet at room temperature $\left(25^{\circ} \mathrm{C}\right)$. The microtubules were monitored under a fluorescence microscope. On addition of ATP, the microtubules started moving with an average velocity of $\sim 480 \pm 21 \mathrm{~nm}$ $\mathrm{s}^{-1}$, and no directional preference of the gliding microtubules was observed just after the ATP addition. Such random movement of microtubules observed just after the addition of ATP was confirmed by measuring the angle of orientation of the microtubules $^{33}$ (ESI Fig. $1 \dagger$ ). Over time, no collective motion was observed, and the microtubules kept randomly moving, which is clear from the fluorescence microscopy images (Fig. 1B, left) and the angle of orientation (ESI Fig. $1 \dagger$ ).

For further confirmation, we quantified the orientation of the microtubules from the fluorescence microscopy images captured at different time intervals after the ATP addition. We calculated the nematic order parameter of the microtubules $(S)$, which is a measure of the orientation of the microtubules. ${ }^{30,31}$ The value of $S$ ranged between 0 and 1 , where $S=0$ or 1 represents random or ordered/aligned orientation of the microtubules, respectively. Our analysis revealed that the nematic order parameter decreased until $\sim 30$ min after ATP addition, and then remained almost constant with time within the investigational period (Fig. 2A). Such a decrease in the nematic order parameter confirmed that no phase transition of microtubules took place from the isotropic to a nematic state. This observation was in agreement with a previous report where no collective motion of microtubules was observed in the absence of depletion force induced by methylcellulose. ${ }^{22}$ The change in the nematic order parameter with time was found to be independent of the concentration of microtubules employed in the gliding assay (Fig. 2A). On increasing the concentration of microtubules in the gliding assay system, the density of microtubules on the kinesin coated surface of the flow cell increased. However, the increased density of the microtubules had no effect on their orientation and failed to result in transition from the isotropic to a nematic state. As reported in literature, snuggling of gliding microtubules is a prerequisite for demonstrating collective motion. ${ }^{20,22}$ Therefore, based on our results, it can be concluded that, in the absence of methylcellulose, even when the density of microtubules was much increased, the behavior of the gliding microtubules was not appreciably changed. As a result, no phase transition was observed. The random motion of microtubules at high concentrations on the kinesins observed in our study was contrary to what was observed on dyneins, ${ }^{20}$ which suggests that the type of motor protein employed in the gliding assay is an important factor in the emergence of the collective motion of microtubules.

Next, we investigated the role of the depletion force in the collective motion of microtubules. We performed the gliding assay of microtubules of varying concentrations as described above. However, the motility of the microtubules was initiated by the addition of ATP buffer containing methylcellulose. Herein, we varied the concentration of methylcellulose over a wide range (0.05-0.3 wt $\%$ ), through which the depletion force among the microtubules was tuned (ESI Fig. $2 \dagger$ ). As discussed above, we monitored the orientation of microtubules over time using fluorescence microscopy and measured the nematic order parameter in each case. When the concentration of methylcellulose was $0.05 \mathrm{wt} \%$, the microtubules at all the concentrations showed a random orientation. Despite the fact that the presence of methylcellulose increases the probability of a snuggling event among the gliding microtubules and favors the emergence of collective motion, ${ }^{22}$ we observed no collective motion of microtubules in the presence of the $0.05 \mathrm{wt} \%$ methylcellulose for any of the employed concentrations of microtubules. The nematic order parameter was found to decrease over time for all the microtubule concentrations, similar to that observed in the absence of methylcellulose (Fig. 2B). On increasing the 
(A) $0.0 \mathrm{wt} \%$

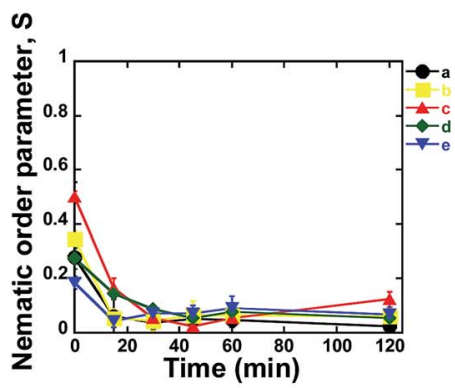

(D) $0.15 w t \%$

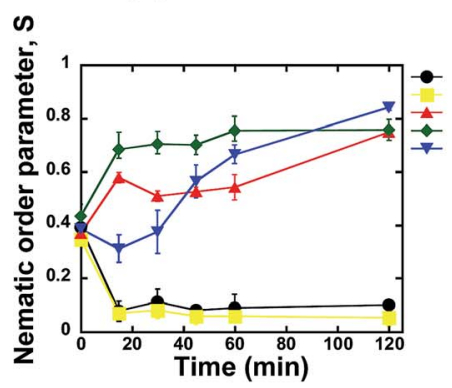

(B) $0.05 w t \%$

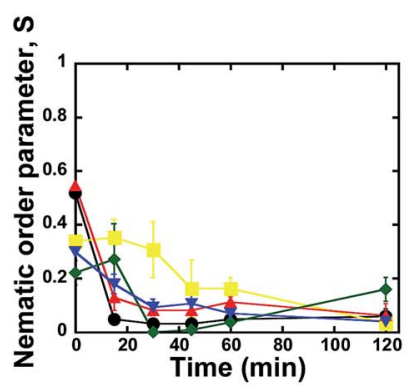

(E) $0.20 w t \%$

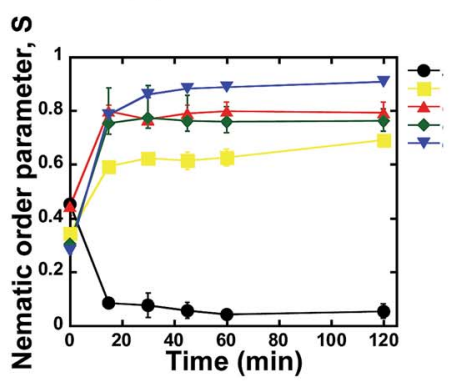

(C) $0.10 w t \%$

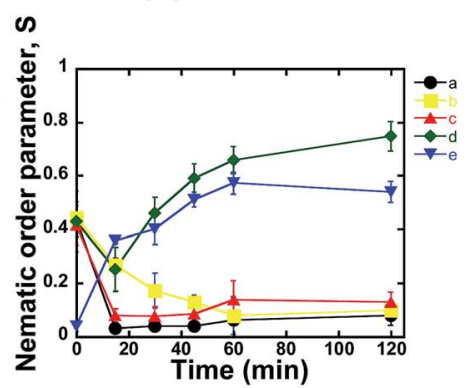

(F) $0.30 w t \%$

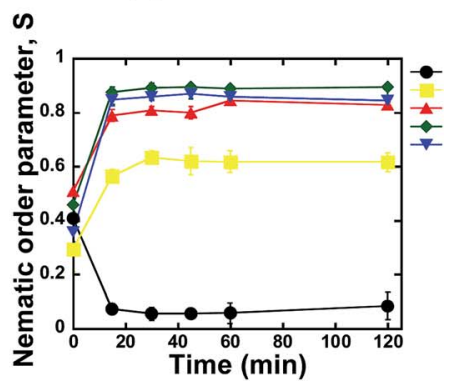

Fig. 2 Change in the orientation of microtubules with time at different methylcellulose and microtubule concentrations. In each case, the concentration of methylcellulose is given above the figure (from 0.0 to $0.3 \mathrm{wt} \%$ ). In these figures, the lines (a, b, c, d, and e) represent the order parameter for $1.0,3.0,5.0,10.0$, and $15.0 \mu \mathrm{M}$ microtubules, respectively.

concentration of methylcellulose to $0.1 \mathrm{wt} \%$, we observed collective motion of microtubules at concentrations of 10 and $15 \mu \mathrm{M}$, but we could not observe the collective motion for the concentrations of 1,3 , and $5 \mu \mathrm{M}$. For the 10 and $15 \mu \mathrm{M}$ microtubules, the nematic order parameter of microtubules gradually increased with time and reached a plateau by $\sim 60 \mathrm{~min}$ after ATP addition (Fig. 2C). At $0.15 \mathrm{wt} \%$ of methylcellulose, collective motion was also observed for $5 \mu \mathrm{M}$ microtubules, in addition to that for 10 and $15 \mu \mathrm{M}$ microtubules. Interestingly we found that the time required to reach the plateau of the nematic order parameter decreased under this condition. As shown in Fig. $2 \mathrm{D}$, it took $\sim 20 \mathrm{~min}$ to form a stable nematic phase of microtubules when the concentration of methylcellulose was $0.15 \mathrm{wt} \%$. On further elevation of the methylcellulose concentration (e.g. at 0.2 and $0.3 \mathrm{wt} \%$ ), collective motion was observed for all the microtubule concentrations employed in this study, except for the concentration of $1 \mu \mathrm{M}$. At these methylcellulose concentrations ( 0.2 and $0.3 \mathrm{wt} \%$ ), no further decrease in the time required to form stable nematic phase was observed (Fig. 2E and F). This observation suggests that even for the high concentrations of microtubules and methylcellulose, a minimum time was required to cause the phase transition of the gliding microtubules from the isotropic to the nematic state.

Based on the above discussed investigations, we summarized our findings, which are shown in Fig. 3A and B. From the summarized results, it is evident that concentration of the microtubules and methylcellulose have profound influences on the kinetics of the collective motion of microtubules on kinesins. For each of the two parameters, a minimum or threshold value was found at or below which no phase transition of microtubules from an isotropic to a nematic state could be observed. Moreover, both the parameters were found to be dependent on each other. The minimum concentration of methylcellulose, which was required to induce the collective motion of microtubules, decreased to lower values when the concentration of microtubules was much higher. On the other hand, a minimum concentration of microtubules was found at or below which no collective motion could be observed even if the concentration of methylcellulose was very high. This minimum concentration of microtubules also depended on the concentration of methylcellulose employed in the gliding assay. The effect of the concentration of microtubules and methylcellulose on the kinetics of phase transition of the microtubules was clearly evident from the heat maps of microtubule orientation at different observation times (Fig. 3A). Initially, just after ATP addition $(0 \mathrm{~min})$, no ordered orientation of microtubules was observed for any of the concentrations of microtubules or methylcellulose. For this observation time, the heat map entirely represents random orientation of the microtubules. Although a little ordered orientation could be detected, this might have been due to transient ordering of the colliding microtubules. At $15 \mathrm{~min}$, the nematic phase could be detected only for relatively higher concentrations of microtubules and methylcellulose. In contrast, for the relatively lower concentrations of microtubules and methylcellulose, the emergence of the collective motion was much slower (e.g., 30 or $45 \mathrm{~min})$. From this stage ( $45 \mathrm{~min})$, a clear separation of the random isotropic and ordered nematic phase could be easily observed from the heat maps, with no further change in microtubule orientation with time. 
(A)

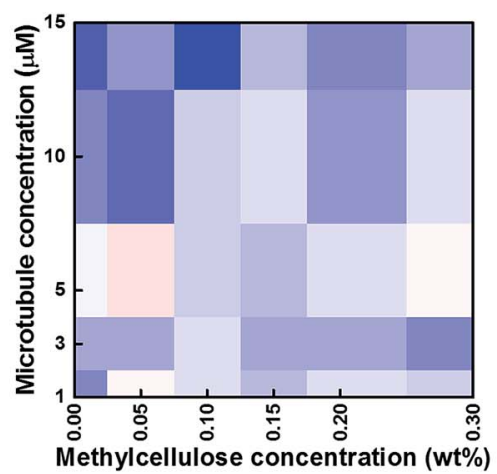

$45 \mathrm{~min}$

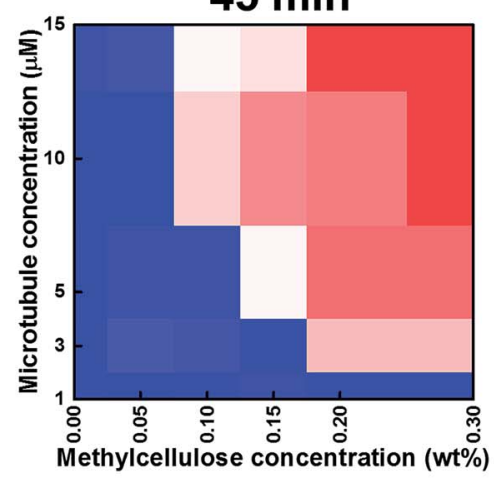

$15 \mathrm{~min}$

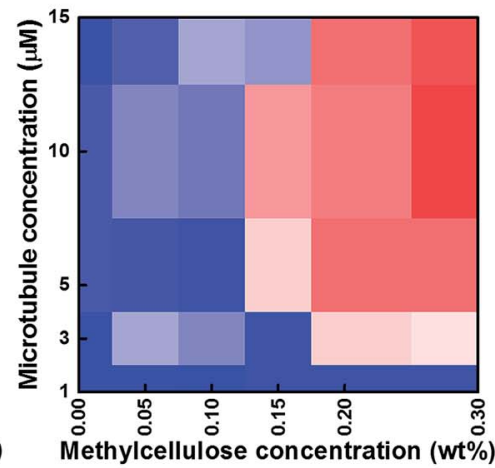

$60 \mathrm{~min}$

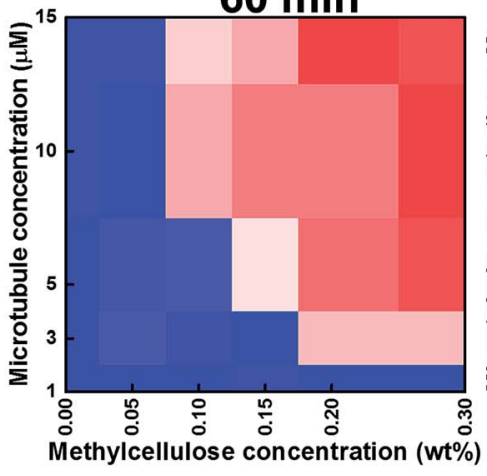

$30 \mathrm{~min}$

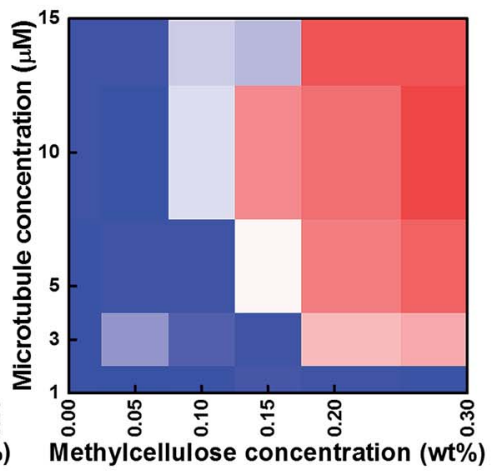

$120 \mathrm{~min}$

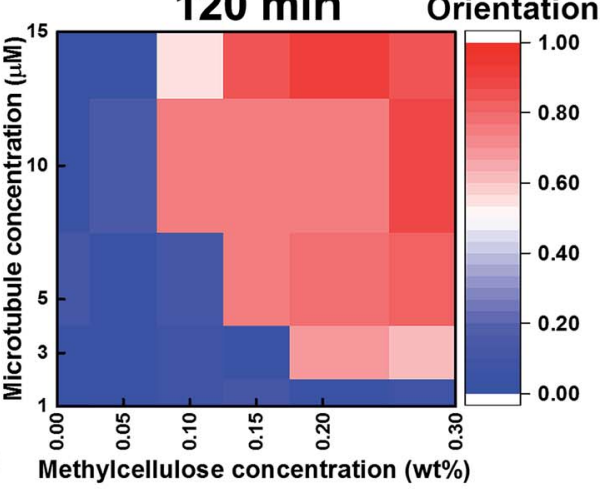

(B)

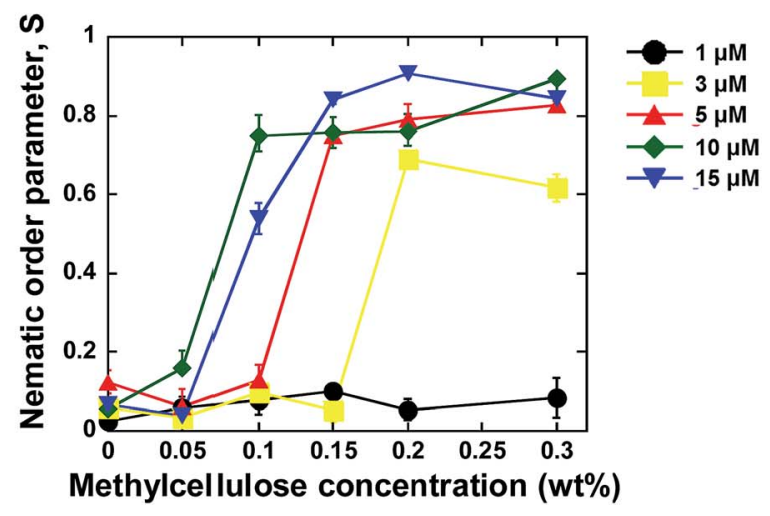

Fig. 3 (A) Heat maps showing the nematic order parameter of microtubules at different observation times as a function of microtubule and methylcellulose concentration. From the heat maps, organization of microtubules and transition of microtubule organization from an isotopic (indicated by blue color) to a nematic phase (indicated by red color) could be observed under different experimental conditions. (B) Change of nematic order parameter with the change in concentration of methylcellulose for different microtubule concentrations, as shown in the legend. The nematic order parameter was calculated after 120 min of ATP addition.

Based on the above discussion, the effect of concentration of microtubules and depletion force on the kinetics of the collective motion of microtubules was clearly evident. To understand the mechanistics in detail, we investigated the behavior of gliding microtubules under different experimental conditions. We quantified the probability of the snuggling and crossing over event of gliding microtubules for different concentrations of methylcellulose. The results, shown in ESI Fig. $3, \dagger$ revealed that methylcellulose had a significant effect on the behavior of the gliding microtubules. In the absence of methylcellulose, the probability of crossing over was much higher compared to that of the snuggling. As reported in the literature, snuggling is the most important behavior of gliding microtubules for demonstrating collective motion. ${ }^{20,22}$ Therefore, the higher probability of crossing over in the absence of methylcellulose might have prevented the microtubules from exhibiting collective motion, even at very high concentrations of the microtubules. This result also implies that the gliding behavior of microtubules was not affected by the concentration of microtubules. Inclusion of the methylcellulose in the gliding assay system (0.05 $\mathrm{wt} \%$ ) was found to increase the probability of snuggling of gliding microtubules. The probability of snuggling kept 


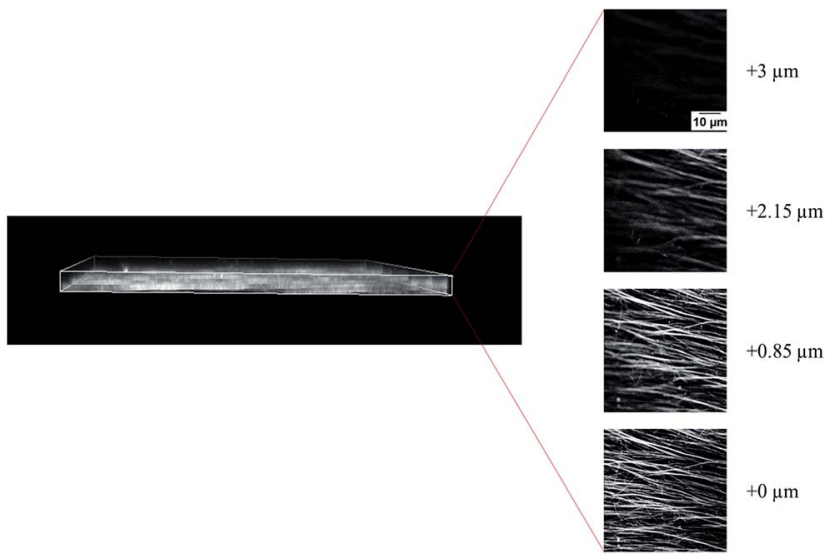

Fig. 4 Two-dimensional structure of the microtubule assemblies in the nematic phase. The images were captured using a confocal laser scanning microscope. The values to the right of the images indicate the height (i.e. focus position along $Z$-axis).

increasing as the concentration of methylcellulose increased and reached a plateau at $0.15 \mathrm{wt} \%$, after which no change was observed on increasing the methylcellulose concentration further. From these results, it can be concluded that methylcellulose expedites the kinetics of the collective motion of microtubules by increasing the probability of snuggling of the gliding microtubules. Herein, it should be mentioned that we observed no considerable effect of methylcellulose on the velocity of microtubules under the present experimental conditions (ESI Fig. $4 \dagger$ ), and the viscosity of the buffer also did not appreciably change on changing the concentration of the methylcellulose within the range studied (ESI Fig. $5 \dagger$ ).

In addition to the kinetic study, we also investigated the structure of the microtubule assemblies in the nematic phase (microtubules in the collective motion) using a confocal laser scanning microscope. The results, shown in Fig. 4, revealed that the nematic phase of the microtubules was a two dimensional structure comprised of overlapping bundles of microtubules. However, changes in the structural organization of microtubule assemblies in the nematic phase in response to the changes in concentration of the microtubules and methylcellulose is unclear at this moment. Understanding this would require a detailed investigation in the future. It is worth noting that, the force that drove the microtubules came from kinesins through consumption of the energy of ATP hydrolysis. The diffusion of kinesin driven microtubules was much faster compared to that driven by thermal forces. ${ }^{34}$ Therefore, in our study, the thermal force induced diffusion had a negligible effect on the movement of microtubules, and the time scale of phase transition of microtubules should be governed by the active movement of microtubules, depletion force and mutual interaction of the dynamic microtubules. ${ }^{22}$

\section{Conclusions}

We have explored the kinetics of the collective motion of microtubules driven by kinesins. We unveiled how the concentration of microtubules and depletion force affect the ensemble behavior of microtubules driven by kinesins. Our results disclosed that both the concentration of microtubules and the depletant (methylcellulose) have crucially important roles in the kinetics of the collective motion of the kinesin driven microtubules. A minimum concentration of microtubules or methylcellulose was required to allow the emergence of collective motion of microtubules and cause a transition from an isotropic to a nematic phase. For low concentrations of methylcellulose (e.g., $<0.05 \mathrm{wt} \%)$, no collective motion of microtubules emerged even for high concentrations of microtubules. On the other hand, even for the relatively higher concentrations of methylcellulose (e.g., $0.3 \mathrm{wt} \%$ ), we observed no collective motion when the concentration of microtubules was low $(1 \mu \mathrm{M})$. A minimum concentration of methylcellulose $(0.1 \mathrm{wt} \%)$ can induce the phase transition of microtubules if the concentration of microtubules is $\geq 10 \mu \mathrm{M}$. Conversely, for the microtubules, a minimum concentration $(3 \mu \mathrm{M})$ was required for the phase transition for any of the concentrations of methylcellulose investigated. Importantly, the time required to cause such a phase transition was also found to be affected by the concentration of microtubules and the methylcellulose employed in the gliding assay. This study offers a better understanding of the collective motion of kinesin driven microtubules, which consequently would be useful in understanding the coordinated behavior of other self-propelled systems in nature. At the same time, this study would be beneficial to expand the nanotechnological applications of biomolecular motor systems. ${ }^{35}$

\section{Acknowledgements}

This study was financially supported by Grant-in-Aid for Scientific Research (B) (JSPS KAKENHI Grant Number JP15H03706) and Grant-in-Aid for Scientific Research on Innovative Areas (JSPS KAKENHI Grant Number JP24104004) from Japan Society for the Promotion of Science (JSPS).

\section{References}

1 T. Vicsek, Nature, 2001, 411, 421.

2 T. Vicsek and A. Zafeiris, Phys. Rep., 2012, 517, 71-140.

3 J. K. Parrish, S. V. Viscido and D. Grünbaum, Biol. Bull., 2002, 202, 296-305.

4 K. Kruse, J. F. Joanny, F. Julicher, J. Prost and K. Sekimoto, Phys. Rev. Lett., 2004, 92, 078101.

5 M. Loose, E. Fischer-Friedrich, J. Ries, K. Kruse and P. Schwille, Science, 2008, 320, 789-792.

6 I. D. Couzin, J. Krause, N. R. Franks and S. A. Levin, Nature, 2005, 433, 513-516.

7 I. H. Riedel, K. Kruse and J. A. Howard, Science, 2005, 309, 300-303.

8 C. Dombrowski, L. Cisneros, S. Chatkaew, R. E. Goldstein and J. O. Kessler, Phys. Rev. Lett., 2004, 93, 098103.

9 A. Czirok, E. Ben-Jacob, I. Cohen and T. Vicsek, Phys. Rev. E: Stat. Phys., Plasmas, Fluids, Relat. Interdiscip. Top., 1996, 54, 1791-1801. 
10 F. J. Nedelec, T. Surrey, A. C. Maggs and S. Leibler, Nature, 1997, 389, 305-308.

11 C. W. Reynolds, Comput Graph., 1987, 21, 25-34.

12 T. Vicsek, A. Czirok, E. Ben-Jacob, I. Cohen and O. Shochet, Phys. Rev. Lett., 1995, 75, 1226-1229.

13 I. D. Couzin, Trends Cognit. Sci., 2008, 13, 36-43.

14 P. Kraikivski, R. Lipowsky and J. Kierfeld, Phys. Rev. Lett., 2006, 96, 258103.

15 H. Levine, W. J. Rappel and I. Cohen, Phys. Rev. E: Stat. Phys., Plasmas, Fluids, Relat. Interdiscip. Top., 2000, 63, 017101.

16 G. Gregoire and H. Chate, Phys. Rev. Lett., 2004, 92, 025702.

17 F. Ginelli, F. Peruani, M. Bär and H. Chate, Phys. Rev. Lett., 2010, 104, 184502.

18 K. H. Nagai, Y. Sumino, R. Montagne, I. S. Aranson and H. Chate, Phys. Rev. Lett., 2015, 114, 168001.

19 V. Schaller, C. Weber, C. Semmrich, E. Frey and A. R. Bausch, Nature, 2010, 467, 73-77.

20 Y. Sumino, K. H. Nagai, Y. Shitaka, Y. Tanaka, K. Yoshikawa, H. Chate and K. Oiwa, Nature, 2012, 483, 448-452.

21 T. Sanchez, D. T. N. Chen, S. J. DeCamp, M. Heymann and Z. Dogic, Nature, 2012, 491, 431-435.

22 D. Inoue, B. Mahmot, A. M. R. Kabir, T. I. Farhana, K. Tokuraku, K. Sada, A. Konagaya and A. Kakugo, Nanoscale, 2015, 7, 18054-18061.

23 G. M. Whitesides and B. Grzybowski, Science, 2002, 295, 2418-2421.
24 M. Fialkowski, K. J. M. Bishop, R. Klajn, S. K. Smoukov, C. J. Campbell and B. A. Grzybowski, J. Phys. Chem. B, 2006, 110, 2482-2496.

25 M. C. Cross and P. C. Hohenberg, Rev. Mod. Phys., 1993, 65, 851-1123.

26 M. Castoldi and A. V. Popov, Protein Expression Purif., 2003, 32, 83-88.

27 R. B. Case, D. W. Pierce, H. B. Nora, L. H. Cynthia and R. D. Vale, Cell, 1997, 90, 959-966.

28 A. M. R. Kabir, D. Inoue, A. Kakugo, A. Kamei and J. P. Gong, Langmuir, 2011, 27, 13659-13668.

29 A. M. R. Kabir, D. Inoue, A. Kakugo, K. Sada and J. P. Gong, Polym. J., 2012, 44, 607-611.

30 K. V. Mardia, and P. E. Jupp, Directional Statistics, John Wiley and Sons Ltd., England, 2000, pp. 57-82.

31 R. Rezakhaniha, A. Agianniotis, J. T. C. Schrauwen, A. Griffa, D. Sage, C. V. C. Bouten, F. N. Van de vosse, M. Unser and N. Stergiopulos, Biomech. Model. Mechanobiol., 2012, 11, 461-473.

32 Y. Jeune-Smith and H. Hess, Soft Matter, 2010, 6, 1778-1784. 33 D. Inoue, T. Nitta, A. M. R. Kabir, K. Sada, J. P. Gong, A. Konagaya and A. Kakugo, Nat. Commun., 2016, 7, 12557. 34 M. Ito, T. Ishiwata, S. Anan, K. Kokado, D. Inoue, A. M. R. Kabir, A. Kakugo and K. Sada, ChemistrySelect, 2016, 1, 5358-5362.

35 W. O. Hancock, Protein-based Nanotechnology: KinesinMicrotubule-driven Systems for Bioanalytical Applications, Wiley-VCH Verlag GmbH \& Co. KGaA, 2007. 